\title{
Weighted energy decay for 1D Dirac equation
}

\author{
E. A. Kopylova
}

Communicated by Sergey Zelik, received Feburary 2, 2011.

\begin{abstract}
We obtain a dispersive long-time decay in weighted energy norms for solutions of the 1D Dirac equation with generic potential. The decay extends the results obtained by Jensen, Kato and Murata for the Schrödinger equations.
\end{abstract}

\section{Contents}

1. Introduction 113

2. Free Dirac equation $\quad 115$

3. Perturbed equation 118

4. Application to asymptotic completeness 124

$\begin{array}{ll}\text { References } & 125\end{array}$

\section{Introduction}

In this paper, we establish a dispersive long time decay for solutions to $1 \mathrm{D}$ Dirac equation

(1.1) $i \dot{\psi}(x, t)=\mathcal{H} \psi(x, t):=i \alpha \psi^{\prime}(x, t)+m \beta \psi(x, t)+\mathcal{V}(x) \psi(x, t), \quad x \in \mathbb{R}, \quad m>0$ in weighted energy norms. Here $\psi(x, t) \in \mathbb{C}^{2}$ for $(x, t) \in \mathbb{R}^{2}$,

$$
\alpha=\left(\begin{array}{ll}
-1 & 0 \\
0 & 1
\end{array}\right), \quad \beta=\left(\begin{array}{ll}
0 & 1 \\
1 & 0
\end{array}\right),
$$

and $\mathcal{V}(x)$ is a given hermitian matrix potential:

$(1.3) \mathcal{V}(x)=\left(\begin{array}{ll}\mathcal{V}_{11}(x) & \mathcal{V}_{12}(x) \\ \mathcal{V}_{21}(x) & \mathcal{V}_{22}(x)\end{array}\right), \mathcal{V}_{11}(x), \mathcal{V}_{22}(x) \in \mathbb{R}, \mathcal{V}_{21}(x)=\overline{\mathcal{V}_{12}(x)}, x \in \mathbb{R}$

1991 Mathematics Subject Classification. 35L10, 34L25, 47A40, 81U05.

Key words and phrases. dispersion, Dirac equation, relativistic equations, resolvent, spectral representation, weighted spaces, continuous spectrum, Born series, convolution, long-time asymptotics.

Supported partly by the FWF, DFG and RFBR grants. 
The matrices $\alpha$ and $\beta$ satisfy the relations

$$
\alpha^{2}=\beta^{2}=I, \quad \alpha \beta+\beta \alpha=0 .
$$

For $s, \sigma \in \mathbb{R}$, let us denote by $H_{\sigma}^{s}=H_{\sigma}^{s}(\mathbb{R})$ the weighted Sobolev spaces [1] , with the finite norms

$$
\|u\|_{H_{\sigma}^{s}}=\left\|\langle x\rangle^{\sigma}\langle d / d x\rangle^{s} u\right\|_{L^{2}(\mathbb{R})}<\infty, \quad\langle x\rangle=\left(1+|x|^{2}\right)^{1 / 2} .
$$

We assume that

$$
\left|\mathcal{V}_{11}(x)\right|+\left|\mathcal{V}_{22}(x)\right|+\left|\mathcal{V}_{12}(x)\right|+\left|\mathcal{V}_{12}^{\prime}(x)\right| \leq C\langle x\rangle^{-\rho}, x \in \mathbb{R}
$$

for some $\rho>5$. Then the multiplication by $\mathcal{V}_{12}$ is bounded operators $H_{s}^{1} \rightarrow H_{s+\rho}^{1}$ for any $s \in \mathbb{R}$.

We restrict ourselves to "nonsingular case" when the truncated resolvent of the operator $\mathcal{H}$ is bounded at the edge points $\lambda= \pm m$ of the continuous spectrum.

Our main result is the following long time decay of the solutions to (1.1): in the nonsingular case

$$
\left\|\mathcal{P}_{c} \psi(t)\right\|_{L_{-\sigma}^{2}}=\mathcal{O}\left(|t|^{-3 / 2}\right), \quad t \rightarrow \pm \infty
$$

for initial data $\psi_{0}=\psi(0) \in L_{\sigma}^{2}:=H_{\sigma}^{0} \otimes \mathbb{C}^{2}$ with $\sigma>5 / 2$ where $\mathcal{P}_{c}$ is a Riesz projection onto the continuous spectrum of the operator $\mathcal{H}$. The decay is desirable for the study of asymptotic stability and scattering for solutions to nonlinear Dirac equations.

Let us comment on previous results in this direction. The decay of type (1.6) in weighted energy norms has been established first by Jensen and Kato [7] for the Schrödinger equation in the dimension $n=3$. The result has been extended to all other dimensions by Jensen and Nenciu $[\mathbf{5}, \mathbf{6}, \mathbf{8}]$, and to more general PDEs of the Schrödinger type by Murata [12]. For the Klein-Gordon equations in the dimensions $n=1,2,3$ the decay of type (1.6) has been proved in [9]-[11] and for $3 \mathrm{D}$ Dirac equation in $[\mathbf{3}]$. For 1D Dirac equation the decay was not obtained before.

Let us comment on our techniques. We extend our approach [9] to the Dirac equation. It is well known that the decay (1.6) violates for the free $1 \mathrm{D}$ Dirac equation corresponding to $\mathcal{V}(x)=0$ when the solutions slow decay, like $\sim t^{-1 / 2}$. The slow decay is caused by the "zero resonance function" $\psi(x)=$ const corresponding to the edge points $\lambda= \pm m$ of the continuous spectrum of the free Dirac operator $\mathcal{H}_{0}=i \alpha \frac{d}{d x}+m \beta$. Hence, the decay (1.6) cannot be deduced by perturbation arguments from the corresponding estimate for the free equation.

The main idea of our approach is a spectral analysis of the "bad" term, with the slow decay $\sim t^{-1 / 2}$. Namely, we show that the bad term does not contribute to the high energy component of solution to the free equation, and the high energy component decays like $t^{-3 / 2}$. Then we prove the decay $\sim t^{-3 / 2}$ for the high energy component of solution to perturbed equation (1.1) using finite Born series and convolutions. For the proof we apply a gauge transformation to obtain a suitable expression for the resolvent of the operator $\mathcal{H}$ via the resolvent of the corresponding "squared Dirac operator" which is a matrix Schrödinger operator with a perturbation. The perturbation does not contain differential operators which allows us to apply the estimates (3.15) obtained in [7] for the Schrödinger operator.

For the low energy component of solution to (1.1), the decay $\sim t^{-3 / 2}$ follows in the nonsingular case by methods $[\mathbf{7}, \mathbf{1 2}]$. 
Our paper is organized as follows. In Section 2 we obtain the time decay for the solution to the free Dirac equation and state the spectral properties of the free resolvent which follow from the corresponding known properties of the free Schrödinger resolvent. In Section 3 we obtain spectral properties of the perturbed resolvent and prove the decay (1.6).

\section{Free Dirac equation}

First, we consider the free Dirac equation:

$$
i \dot{\psi}(x, t)=\mathcal{H}_{0} \psi(x, t):=i \alpha \psi^{\prime}(x, t)+m \beta \psi(x, t) .
$$

Denote by $\mathcal{U}(t): \psi(\cdot, 0) \rightarrow \psi(\cdot, t)$ the dynamical group of equation (2.1). It is a strongly continuous group in $L^{2}:=L^{2}(\mathbb{R}) \otimes \mathbb{C}^{2}$. The group is unitary that corresponds to the charge conservation.

2.1. Spectral properties. We state spectral properties of the free dynamical group $\mathcal{U}(t)$ applying known results of $[\mathbf{1}, \mathbf{1 2}]$ which concern the spectral properties of the free Schrödinger dynamical group. For $t>0$ and $\psi_{0}=\psi(0) \in L^{2}$, the solution $\psi(t)$ to free equation (2.1) admits the spectral Fourier-Laplace representation

$$
\theta(t) \psi(t)=\frac{1}{2 \pi i} \int_{\mathbb{R}} e^{-i(\omega+i \varepsilon) t} \mathcal{R}_{0}(\omega+i \varepsilon) \psi_{0} d \omega, t \in \mathbb{R}
$$

with any $\varepsilon>0$ where $\theta(t)$ is the Heaviside function, $\mathcal{R}_{0}(\omega)=\left(\mathcal{H}_{0}-\omega\right)^{-1}$ is the resolvent of the operator $\mathcal{H}_{0}$. The representation follows from the stationary equation $\omega \tilde{\psi}^{+}(\omega)=\mathcal{H}_{0} \tilde{\psi}^{+}(\omega)+i \psi_{0}$ for the Fourier-Laplace transform $\tilde{\psi}^{+}(\omega):=$ $\int_{\mathbb{R}} \theta(t) e^{i \omega t} \psi(t) d t$, where $\omega \in \mathbb{C}^{+}:=\{\operatorname{Im} \omega>0\}$. The solution $\psi(t)$ is a continuous bounded function of $t \in \mathbb{R}$ with the values in $L^{2}$. Hence, $\tilde{\psi}^{+}(\omega)=-i \mathcal{R}_{0}(\omega) \psi_{0}$ is an analytic function of $\omega \in \mathbb{C}^{+}$with the values in $L^{2}$, bounded for $\omega \in \mathbb{R}+i \varepsilon$. Therefore, integral (2.2) converges in the sense of distributions of $t \in \mathbb{R}$ with the values in $L^{2}$. Similarly to $(2.2)$,

$$
\theta(-t) \psi(t)=-\frac{1}{2 \pi i} \int_{\mathbb{R}} e^{-i(\omega-i \varepsilon) t} \mathcal{R}_{0}(\omega-i \varepsilon) \psi_{0} d \omega, t \in \mathbb{R} .
$$

The resolvent $\mathcal{R}_{0}(\omega)$ can be expressed in terms of the resolvent $R_{0}(\zeta)=\left(-\partial_{x}^{2}-\zeta\right)^{-1}$ of the free Schrödinger operator. Indeed, (1.4) implies

$$
\left(\mathcal{H}_{0}-\omega\right)\left(\mathcal{H}_{0}+\omega\right)=\left(i \alpha \partial_{x}+m \beta-\omega\right)\left(i \alpha \partial_{x}+m \beta+\omega\right)=\left(-\partial_{x}^{2}+m^{2}-\omega^{2}\right) .
$$

Therefore,

$$
\mathcal{R}_{0}(\omega)=\left(i \alpha \partial_{x}+m \beta+\omega\right) R_{0}\left(\omega^{2}-m^{2}\right)
$$

where $R_{0}(\zeta)$ is the operator with the integral kernel

$$
R_{0}(\zeta, x-y)=-\frac{\exp (i \sqrt{\zeta}|x-y|)}{2 i \sqrt{\zeta}}, \quad \zeta \in \mathbb{C} \backslash[0, \infty), \quad \operatorname{Im} \zeta^{1 / 2}>0 .
$$

Denote by $\mathcal{L}\left(B_{1}, B_{2}\right)$ the Banach space of bounded linear operators from a Banach space $B_{1}$ to a Banach space $B_{2}$. Explicit formula (2.6) obviously implies the properties of $R_{0}(\zeta)$ (cf. $\left.[\mathbf{1}, \mathbf{1 2}]\right)$ : 
i) $R_{0}(\zeta)$ is an analytic function of $\zeta \in \mathbb{C} \backslash[0, \infty)$ with the values in $\mathcal{L}\left(H^{0}, H^{2}\right)$;

ii) For $\zeta>0$, the convergence holds

$$
R_{0}(\zeta \pm i \varepsilon) \rightarrow R_{0}(\zeta \pm i 0), \quad \varepsilon \rightarrow 0+
$$

in $\mathcal{L}\left(H_{\sigma}^{0}, H_{-\sigma}^{2}\right)$ with $\sigma>1 / 2$, uniformly in $\zeta \geq r$ for any $r>0$.

iii) In $\mathcal{L}\left(H_{\sigma}^{0} ; H_{-\sigma}^{2}\right)$ with $\sigma>5 / 2$ the asymptotics hold

$$
R_{0}(\zeta)=A_{0} \zeta^{-1 / 2}+A_{1}+\mathcal{O}\left(\zeta^{1 / 2}\right), \quad R_{0}^{\prime}(\zeta)=-\frac{1}{2} A_{0} \zeta^{-3 / 2}+\mathcal{O}\left(\zeta^{-1 / 2}\right),
$$

as $\zeta \rightarrow 0, \zeta \in \mathbb{C} \backslash[0, \infty)$. Here

$$
\begin{aligned}
& A_{0}=\mathrm{Op}\left[\frac{i}{2}\right] \in \mathcal{L}\left(H_{\sigma}^{0} ; H_{-\sigma}^{2}\right), \sigma>1 / 2, \\
& A_{1}=\mathrm{Op}\left[-\frac{1}{2}|x-y|\right] \in \mathcal{L}\left(H_{\sigma}^{0} ; H_{-\sigma}^{2}\right), \sigma>3 / 2 .
\end{aligned}
$$

iv) For $l=0,1, k=0,1,2, \ldots$ and $\sigma>1 / 2+k$ the asymptotics hold

$$
\left\|R_{0}^{(k)}(\zeta)\right\|_{\mathcal{L}\left(H_{\sigma}^{0}, H_{-\sigma}^{l}\right)}=\mathcal{O}\left(|\zeta|^{-\frac{1-l+k}{2}}\right), \quad \zeta \rightarrow \infty, \quad \zeta \in \mathbb{C} \backslash(0, \infty) .
$$

Let us denote $\Gamma:=(-\infty,-m) \cup(m, \infty)$, The properties i) - iv) and formula (2.5) imply

Lemma 2.1. i) The resolvent $\mathcal{R}_{0}(\omega)$ is an analytic function of $\omega \in \mathbb{C} \backslash \bar{\Gamma}$ with the values in $\mathcal{L}\left(L^{2}, L^{2}\right)$.

ii) For $\omega \in \Gamma$, the convergence holds

$$
\mathcal{R}_{0}(\omega \pm i \varepsilon) \rightarrow \mathcal{R}_{0}(\omega \pm i 0), \varepsilon \rightarrow 0+
$$

in $\mathcal{L}\left(L_{\sigma}^{2}, L_{-\sigma}^{2}\right)$ with $\sigma>1 / 2$, uniformly in $|\omega| \geq m+r$ for any $r>0$.

iii) In $\mathcal{L}\left(L_{\sigma}^{2} ; L_{-\sigma}^{2}\right)$ with $\sigma>5 / 2$ the asymptotics hold

(2.12)

$$
\begin{aligned}
& \mathcal{R}_{0}(\omega)=\mathcal{A}_{0}^{ \pm}(\omega \mp m)^{-1 / 2}+\mathcal{A}_{1}^{ \pm}+\mathcal{O}\left((\omega \mp m)^{1 / 2}\right) \\
& \mathcal{R}_{0}^{\prime}(\omega)=-\frac{1}{2} \mathcal{A}_{0}^{ \pm}(\omega \mp m)^{-3 / 2}+\mathcal{O}\left((\omega \mp m)^{-1 / 2}\right) \\
& \mathcal{R}_{0}^{\prime \prime}(\omega)=\mathcal{O}\left((\omega \mp m)^{-3 / 2}\right)
\end{aligned} \mid \omega \rightarrow \pm m, \quad \omega \in \mathbb{C} \backslash \bar{\Gamma} .
$$

Here

$$
\mathcal{A}_{0}^{ \pm}=\mathrm{Op}\left[\frac{i \sqrt{m}}{\sqrt{ \pm 2}}\left(\begin{array}{cc} 
\pm 1 & 1 \\
1 & \pm 1
\end{array}\right)\right] \in \mathcal{L}\left(L_{\sigma}^{2} ; L_{-\sigma}^{2}\right), \sigma>1 / 2,
$$

$$
\begin{aligned}
\mathcal{A}_{1}^{ \pm}= & \mathrm{Op}\left[\frac{-m|x-y|}{2}\left(\begin{array}{cc} 
\pm 1 & 1 \\
1 & \pm 1
\end{array}\right)-\frac{1}{2}\left(\begin{array}{cc}
\operatorname{sgn}(x-y) & 0 \\
0 & \operatorname{sgn}(x-y)
\end{array}\right)\right] \\
& \in \mathcal{L}\left(L_{\sigma}^{2} ; L_{-\sigma}^{2}\right), \sigma>3 / 2
\end{aligned}
$$

iv) For $k=0,1,2, \ldots$ and $\sigma>1 / 2+k$ the asymptotics hold

$$
\left\|\mathcal{R}_{0}^{(k)}(\omega)\right\|_{\mathcal{L}\left(L_{\sigma}^{2}, L_{-\sigma}^{2}\right)}=\mathcal{O}(1) \quad \omega \rightarrow \infty, \quad \omega \in \mathbb{C} \backslash \Gamma
$$


Corollary 2.2. For $t \in \mathbb{R}$ and $\psi_{0} \in L_{\sigma}^{2}$ with $\sigma>1 / 2$, the group $\mathcal{U}(t)$ admits the integral representation

$$
\mathcal{U}(t) \psi_{0}=\frac{1}{2 \pi i} \int_{\Gamma} e^{-i \omega t}\left[\mathcal{R}_{0}(\omega+i 0)-\mathcal{R}_{0}(\omega-i 0)\right] \psi_{0} d \omega
$$

where the integral converges in the sense of distributions of $t \in \mathbb{R}$ with the values in $L_{-\sigma}^{2}$.

Proof. Summing up representations (2.2) and (2.3), and sending $\varepsilon \rightarrow 0+$, we obtain (2.16) by the Cauchy theorem and lemma 2.1 .

2.2. Time decay. Here we prove time decay (1.6) for the free Dirac equation (2.1). Let $G(t)=\mathrm{Op}[G(x-y, t)]$, where $G(x, t)$ is the fundamental solution to the Klein-Gordon operator $\partial_{t}^{2}-\partial_{x}^{2}+m^{2}$ :

$$
G(x, t)=\frac{1}{2} \theta(t-|x|) J_{0}\left(m \sqrt{t^{2}-x^{2}}\right) .
$$

where $J_{0}$ is the Bessel function. Since

$$
\left(\partial_{t}+\alpha \partial_{x}-i m \beta\right)\left(\partial_{t}-\alpha \partial_{x}+i m \beta\right)=\partial_{t}^{2}-\partial_{x}^{2}+m^{2}
$$

we have for the integral kernel $\mathcal{U}(x-y, t)$ of the operator $\mathcal{U}(t)$

$$
\mathcal{U}(x, t)=\left(\partial_{t}+\alpha \partial_{x}-i m \beta\right) G(x, t) .
$$

The asymptotics of the Bessel functions [13] imply

$$
G(x, t) \sim t^{-1 / 2}, \quad \partial_{t} G(x, t) \sim t^{-1 / 2}, \quad \partial_{x} G(x, t) \sim t^{-1 / 2}, \quad x \in \mathbb{R}, \quad t \rightarrow \infty
$$

Hence (2.17) implies

$$
\mathcal{U}(x, t) \sim t^{-1 / 2}, \quad x \in \mathbb{R}, \quad t \rightarrow \infty
$$

and then the free group $\mathcal{U}(t)$ decays like $t^{-1 / 2}$.

The operator $G(t)$ admits the integral representation similar to (2.16):

$$
G(t) \psi_{0}=\frac{1}{2 \pi} \int_{\Gamma} e^{-i \omega t}\left[R_{0}\left((\omega+i 0)^{2}-m^{2}\right)-R_{0}\left((\omega-i 0)^{2}-m^{2}\right)\right] \psi_{0} d \omega, \quad t \in \mathbb{R}
$$

for $\psi_{0} \in L_{\sigma}^{2}$ with $\sigma>1 / 2$. Let us introduce the low energy and high energy components of $G(t)$ and $\mathcal{U}(t)$ :

$$
\begin{aligned}
& \text { (2.19) } G_{l}(t)=\frac{1}{2 \pi} \int_{\Gamma} e^{-i \omega t} l(\omega)\left[R_{0}\left((\omega+i 0)^{2}-m^{2}\right)-R_{0}\left((\omega-i 0)^{2}-m^{2}\right)\right] d \omega \\
& (2.20) \quad G_{h}(t)=\frac{1}{2 \pi} \int_{\Gamma} e^{-i \omega t} h(\omega)\left[R_{0}\left((\omega+i 0)^{2}-m^{2}\right)-R_{0}\left((\omega-i 0)^{2}-m^{2}\right)\right] d \omega \\
& (2.21) \quad \mathcal{U}_{l}(t)=\frac{1}{2 \pi i} \int_{\Gamma} e^{-i \omega t} l(\omega)\left[\mathcal{R}_{0}(\omega+i 0)-\mathcal{R}_{0}(\omega-i 0)\right] d \omega \\
& \text { (2.22) } \mathcal{U}_{h}(t)=\frac{1}{2 \pi i} \int_{\Gamma} e^{-i \omega t} h(\omega)\left[\mathcal{R}_{0}(\omega+i 0)-\mathcal{R}_{0}(\omega-i 0)\right] d \omega
\end{aligned}
$$

where $l(\omega) \in C_{0}^{\infty}(\mathbb{R})$ is an even function, supp $l \in[-m-2 \varepsilon, m+2 \varepsilon], l(\omega)=1$ if $|\omega| \leq m+\varepsilon$, and $h(\omega)=1-l(\omega)$. In [9] we have proved that $G_{h}(t)$ decays like $t^{-3 / 2}$. Here we will prove that $\mathcal{U}_{h}(t)$ also decays like $t^{-3 / 2}$. 
Theorem 2.3. Let $\sigma>5 / 2$. Then the decay holds

$$
\left\|\mathcal{U}_{h}(t)\right\|_{\mathcal{L}\left(L_{\sigma}^{2} ; L_{-\sigma}^{2}\right)} \leq C(1+|t|)^{-3 / 2}, \quad t \in \mathbb{R} .
$$

Proof. First, taking into account (2.17), (2.20), (2.22) we obtain that

$$
\mathcal{U}_{h}(t)=\left(\partial_{t}+\alpha \partial_{x}-i m \beta\right) G_{h}(t) .
$$

Further, [9, Theorem 2.7] implies that for $\sigma>5 / 2$

$$
\left.\left\|G_{h}(t)\right\|_{\mathcal{L}\left(H_{\sigma}^{0} ; H_{-\sigma}^{1}\right)}+\left\|\partial_{t} G_{h}(t)\right\|_{\mathcal{L}\left(L_{\sigma}^{2} ; L_{-\sigma}^{2}\right)} \leq C(1+|t|)^{-3 / 2}\right), \quad t \in \mathbb{R} .
$$

Then (2.24) and (2.25) imply (2.23).

\section{Perturbed equation}

To prove a long time decay for the perturbed equation, we first establish the spectral properties of its generator $\mathcal{H}$.

3.1. Spectral properties. Similarly to [12, formula (3.1)], let us introduce a generalized eigenspaces $\mathbf{M}^{ \pm}$of the operator $\mathcal{H}$ :

$$
\mathbf{M}^{ \pm}=\left\{\psi \in H_{-1 / 2-0}^{1}:\left(1+\mathcal{A}_{1}^{ \pm} \mathcal{V}\right) \psi \in \Re\left(\mathcal{A}_{0}^{ \pm}\right), \quad \mathcal{A}_{0}^{ \pm} \mathcal{V} \psi=0\right\}
$$

where $\mathcal{A}_{0}^{ \pm}$and $\mathcal{A}_{1}^{ \pm}$are defined in (2.14), and $\Re\left(\mathcal{A}_{0}^{ \pm}\right)$denotes the range of $\mathcal{A}_{0}^{ \pm}$. Below we assume that

$$
\mathbf{M}^{ \pm}=0
$$

Denote by $\mathcal{R}(\omega)=(\mathcal{H}-\omega)^{-1}, \omega \in \mathbb{C} \backslash \Gamma$, the resolvents of the operators $\mathcal{H}$. Next lemma is the vector version of $[\mathbf{1 2}$, Theorem 7.2].

Lemma 3.1. Let conditions (1.5) and (3.1) hold. Then the families $\{\mathcal{R}( \pm m+$ $\varepsilon): \pm m+\varepsilon \in \mathbb{C} \backslash \bar{\Gamma},|\varepsilon|<\delta\}$ are bounded in the operator norm of $\mathcal{L}\left(L_{\sigma}^{2}, L_{-\sigma}^{2}\right)$ for any $\sigma>3 / 2$ and sufficiently small $\delta$.

Asymptotics (2.12) and lemma 3.1 imply

Proposition 3.2. Let the conditions (1.5) and (3.1) hold. Then the asymptotics hold

$$
\begin{aligned}
& \mathcal{R}(\omega)-\mathcal{R}( \pm m)=\mathcal{O}\left(|\omega \mp m|^{1 / 2}\right) \\
& \mathcal{R}^{\prime}(\omega)=\mathcal{O}\left(|\omega \mp m|^{-1 / 2}\right) \\
& \mathcal{R}^{\prime \prime}(\omega)=\mathcal{O}\left(|\omega \mp m|^{-3 / 2}\right)
\end{aligned} \mid \omega \rightarrow \pm m, \quad \omega \in \mathbb{C} \backslash \bar{\Gamma}
$$

in $\mathcal{L}\left(L_{\sigma}^{2}, L_{-\sigma}^{2}\right)$ with $\sigma>5 / 2$.

Proof. Lemma 3.1 implies that for any $\sigma>3 / 2$ the operators $\left(1+\mathcal{R}_{0}(\omega) \mathcal{V}\right)^{-1}=$ $1-\mathcal{R}(\omega) \mathcal{V}$ and $\left(1+\mathcal{V} \mathcal{R}_{0}(\omega)\right)^{-1}=1-\mathcal{V} \mathcal{R}(\omega)$ are bounded in $\mathcal{L}\left(L_{-\sigma}^{2}, L_{-\sigma}^{2}\right)$ and in $\mathcal{L}\left(L_{\sigma}^{2}, L_{\sigma}^{2}\right)$ respectively for $|\omega \mp m|<\delta, \omega \in \mathbb{C} \backslash \bar{\Gamma}$ with $\delta$ sufficiently small. Asymptotics (2.12) imply

$$
\begin{array}{l|l}
\mathcal{R}(\omega)=\left(1+\mathcal{R}_{0}(\omega) \mathcal{V}\right)^{-1} \mathcal{R}_{0}(\omega) & \\
=\left(1+\mathcal{R}_{0}(\omega) \mathcal{V}\right)^{-1}\left(\mathcal{A}_{0}^{ \pm} \frac{1}{\sqrt{\omega \mp m}}+\mathcal{O}(1)\right) & \omega \rightarrow \pm m, \omega \in \mathbb{C} \backslash \bar{\Gamma} \\
\mathcal{R}(\omega)=\mathcal{R}_{0}(\omega)\left(1+\mathcal{V} \mathcal{R}_{0}(\omega)\right)^{-1} & \\
=\left(A_{0}^{ \pm} \frac{1}{\sqrt{\omega \mp m}}+\mathcal{O}(1)\right)\left(1+\mathcal{V} \mathcal{R}_{0}(\omega)\right)^{-1} &
\end{array}
$$


in $\mathcal{L}\left(L_{\sigma}^{2}, L_{-\sigma}^{2}\right)$ with $\sigma>3 / 2$. Hence, the boundedness of $\mathcal{R}(\omega),\left(1+\mathcal{R}_{0}(\omega) \mathcal{V}\right)^{-1}$ and $\left(1+\mathcal{V} \mathcal{R}_{0}(\omega)\right)^{-1}$ at the points $\omega= \pm m$ in the corresponding norms imply that

$$
\begin{aligned}
& \left(1+\mathcal{R}_{0}(\omega) \mathcal{V}\right)^{-1} \mathcal{A}_{0}^{ \pm}=\mathcal{O}(\sqrt{\omega \mp m}), \\
& \mathcal{A}_{0}^{ \pm}\left(1+\mathcal{V} \mathcal{R}_{0}(\omega)\right)^{-1}=\mathcal{O}(\sqrt{\omega \mp m}), \omega \rightarrow 0, \omega \in \mathbb{C} \backslash \bar{\Gamma}
\end{aligned}
$$

in $\mathcal{L}\left(L_{\sigma}^{2}, L_{-\sigma}^{2}\right)$ with $\sigma>3 / 2$. Therefore,

(3.4) $\left\|\left(1+\mathcal{R}_{0}(\omega) V\right)^{-1}[1]\right\|_{L_{-\sigma}^{2}}=\mathcal{O}(\sqrt{\omega \mp m}), \quad \omega \rightarrow \pm m, \quad \omega \in \mathbb{C} \backslash \bar{\Gamma}, \quad \sigma>3 / 2$

and for any $f \in L_{\sigma}^{2}$ with $\sigma>3 / 2$

$$
\int\left[\left(1+\mathcal{V} \mathcal{R}_{0}(\omega)\right)^{-1} f\right](x) d x=\mathcal{O}(\sqrt{\omega \mp m}), \quad \omega \rightarrow \pm m, \quad \omega \in \mathbb{C} \backslash \bar{\Gamma}, \quad \sigma>3 / 2 .
$$

Taking into account the identities

$\mathcal{R}^{\prime}=\left(1+\mathcal{R}_{0} \mathcal{V}\right)^{-1} \mathcal{R}_{0}^{\prime}\left(1+\mathcal{V} \mathcal{R}_{0}\right)^{-1}, \quad \mathcal{R}^{\prime \prime}=\left[\left(1+\mathcal{R}_{0} \mathcal{V}\right)^{-1} \mathcal{R}_{0}^{\prime \prime}-2 \mathcal{R}^{\prime} \mathcal{V} \mathcal{R}_{0}^{\prime}\right]\left(1+\mathcal{V} \mathcal{R}_{0}\right)^{-1}$ we obtain from (3.4)-(3.5) asymptotics $(3.2)$ for $\mathcal{R}^{\prime}(\omega)$ and $\mathcal{R}^{\prime \prime}(\omega)$. Finally, asymptotics (3.2) for $\mathcal{R}(\omega)$ follow by integration of asymptotics $(3.2)$ for $\mathcal{R}^{\prime}(\omega)$.

To obtain other properties of $\mathcal{R}(\omega)$, we express $\mathcal{R}(\omega)$ in the resolvent of a matrix Schrödinger operator. First, we introduce the operator $\tilde{\mathcal{H}}$ similar to $\mathcal{H}$ with matrix potential $\tilde{\mathcal{V}}(x)$ satisfying $\tilde{\mathcal{V}}_{11}(x)=\tilde{\mathcal{V}}_{22}(x)=0$. Namely, let us introduce the matrix of gauge transformation

$$
C=\left(\begin{array}{cc}
\exp \left(-i \int_{-\infty}^{x} \mathcal{V}_{11}(y) d y\right) & 0 \\
0 & \exp \left(i \int_{-\infty}^{x} \mathcal{V}_{22}(y) d y\right)
\end{array}\right)
$$

This matrix function is bounded by conditions (1.3), and

$$
C^{-1} \mathcal{H} C=\tilde{\mathcal{H}}
$$

where

$$
\tilde{\mathcal{H}}:=i \alpha \partial_{x}+\tilde{\mathcal{V}}, \quad \tilde{\mathcal{V}}=\left(\begin{array}{cc}
0 & \tilde{\mathcal{V}}_{12} \\
\tilde{\mathcal{V}}_{21} & 0
\end{array}\right)
$$

with

$$
\tilde{\mathcal{V}}_{12}(x)=\exp \left(i \int_{-\infty}^{x}\left(\mathcal{V}_{11}(y)+\mathcal{V}_{22}(y)\right) d y\right)\left(\mathcal{V}_{12}(x)+m\right), \quad \tilde{\mathcal{V}}_{21}(x)=\overline{\tilde{\mathcal{V}}_{12}(x)}
$$

By (3.7), the spectral properties of $\mathcal{H}$ and $\tilde{\mathcal{H}}$ are identical. Further, we have

$$
\begin{aligned}
& (\tilde{\mathcal{H}}-\omega)(\tilde{\mathcal{H}}+\omega)=\left(i \alpha \partial_{x}+\tilde{\mathcal{V}}(x)-\omega\right)\left(i \alpha \partial_{x}+\tilde{\mathcal{V}}(x)+\omega\right) \\
= & \left(\begin{array}{cc}
-\partial_{x}^{2}+m^{2}-\omega^{2}+2 m \operatorname{Re} \mathcal{V}_{12}+\left|\mathcal{V}_{12}\right|^{2} & -i \tilde{\mathcal{V}}_{12}^{\prime} \\
i \tilde{\mathcal{V}}_{21}^{\prime} & -\partial_{x}^{2}+m^{2}-\omega^{2}+2 m \operatorname{Re} \mathcal{V}_{12}+\left|\mathcal{V}_{12}\right|^{2}
\end{array}\right)
\end{aligned}
$$

(3.10) $=\mathbf{H}-\left(\omega^{2}-m^{2}\right)$.

Here $\mathbf{H}=\mathbf{H}_{0}+V$ is the matrix Schrödinger operator with

$$
\mathbf{H}_{0}=\left(\begin{array}{cc}
-\partial_{x}^{2} & 0 \\
0 & -\partial_{x}^{2}
\end{array}\right), \quad V=\left(\begin{array}{cc}
2 m \operatorname{Re} \mathcal{V}_{12}+\left|\mathcal{V}_{12}\right|^{2} & -i \tilde{\mathcal{V}}_{12}^{\prime} \\
i \tilde{\mathcal{V}}_{21}^{\prime} & 2 m \operatorname{Re} \mathcal{V}_{12}+\left|\mathcal{V}_{12}\right|^{2}
\end{array}\right)
$$


Remark 3.3. Due to (1.3), the perturbation $V$ is hermitian and does not contain differential operators. This fact is essential for obtaining high energy decay (3.31), (3.33), and (3.35).

Formula (3.10) implies the following representation for the resolvent $\tilde{\mathcal{R}}(\omega)=$ $(\tilde{\mathcal{H}}-\omega)^{-1}$ :

$$
\tilde{\mathcal{R}}(\omega)=(\tilde{\mathcal{H}}+\omega) R\left(\omega^{2}-m^{2}\right)=\left(i \alpha \partial_{x}+\tilde{\mathcal{V}}(x)+\omega\right) R\left(\omega^{2}-m^{2}\right)
$$

where $R(\zeta)=(\mathbf{H}-\zeta)^{-1}, \zeta \in \mathbb{C} \backslash[0, \infty)$, the resolvent of the operators $\mathbf{H}$.

Due to (3.7), we have $\tilde{\mathcal{R}}(\omega)=C \mathcal{R}(\omega) C^{-1}$. The resolvent $\tilde{\mathcal{R}}(\omega)$ admits the low energy asymptotics of type (3.2) since matrix function (3.6) is bounded. Namely

$$
\begin{array}{l|l}
\tilde{\mathcal{R}}(\omega)-\tilde{\mathcal{R}}( \pm m)=\mathcal{O}\left(|\omega \mp m|^{1 / 2}\right) & \\
\tilde{\mathcal{R}}^{\prime}(\omega)=\mathcal{O}\left(|\omega \mp m|^{-1 / 2}\right) & \omega \rightarrow \pm m, \quad \omega \in \mathbb{C} \backslash \bar{\Gamma} \\
\tilde{\mathcal{R}}^{\prime \prime}(\omega)=\mathcal{O}\left(|\omega \mp m|^{-3 / 2}\right) &
\end{array}
$$

in $\mathcal{L}\left(L_{\sigma}^{2}, L_{-\sigma}^{2}\right)$ with $\sigma>5 / 2$.

Below we need a limiting absorption principle and a high energy decay for the resolvent $\tilde{\mathcal{R}}(\omega)$. First, we obtain these properties for the resolvent $R(\zeta)$ of the matrix Schrödinger operator $\mathbf{H}$.

Lemma 3.4. Let conditions (1.5) and (3.1) hold. Then i) $R(\zeta)$ is a meromorphic function of $\zeta \in \mathbb{C} \backslash[0, \infty)$.

ii) For $\zeta>0$, the convergence (limiting absorption principle) holds

$$
R(\zeta \pm i \varepsilon) \rightarrow R(\zeta \pm i 0), \quad \varepsilon \rightarrow 0+
$$

in $\mathcal{L}\left(H_{\sigma}^{0}, H_{-\sigma}^{2}\right)$ with $\sigma>1 / 2$.

iii) For $l=0,1$, the asymptotics hold

$$
\left\|R_{j}^{(k)}(\zeta)\right\|_{\mathcal{L}\left(H_{\sigma}^{0}, H_{-\sigma}^{l}\right)}=\mathcal{O}\left(|\zeta|^{-\frac{1-l+k}{2}}\right), \quad \zeta \rightarrow \infty, \quad \zeta \in \mathbb{C} \backslash[0, \infty)
$$

with $\sigma>1 / 2+k$ for $k=0,1,2$.

Proof. Step i) The statement i) follows from lemma 2.1-i), the Born splitting

$$
R(\zeta)=R_{0}(\zeta)\left(1+V R_{0}(\zeta)\right)^{-1}
$$

and the Gohberg-Bleher theorem $[\mathbf{2}, \mathbf{4}]$ since the operators $V R_{0}(\zeta)$ are compact operators in $L^{2}$ for $\zeta \in \mathbb{C} \backslash[0, \infty)$.

Step ii) Convergence (3.14) follows from a vector version of Agmon's theorem [1, Theorem 3.3 and Lemma 4.2] taking into account the absence of embedded eigenvalues which follows from the theory of ordinary differential equations since $V \in L^{1}$. Step iii) The asymptotics (3.15) follows from (2.10) by a vector version of $[\mathbf{7}$, Theorem 9.2].

Lemma 3.4 and formula (3.12) imply

LEMMA 3.5. Let conditions (1.5) and (3.1) hold. Then

i) $\tilde{\mathcal{R}}(\omega)$ is a meromorphic function of $\omega \in \mathbb{C} \backslash \bar{\Gamma}$ with the values in $\mathcal{L}\left(L^{2}, L^{2}\right)$;

ii) For $\omega \in \Gamma$, the convergence (limiting absorption principle) holds

$$
\tilde{\mathcal{R}}(\omega \pm i \varepsilon) \rightarrow \tilde{\mathcal{R}}(\omega \pm i 0), \quad \varepsilon \rightarrow 0+
$$

in $\mathcal{L}\left(L_{\sigma}^{2}, L_{-\sigma}^{2}\right)$ with $\sigma>1 / 2$;

iii) For $k=0,1,2$ and $\sigma>1 / 2+k$ the asymptotics hold

$$
\left\|\tilde{\mathcal{R}}^{(k)}(\omega)\right\|_{\mathcal{L}\left(L_{\sigma}^{2}, L_{-\sigma}^{2}\right)}=\mathcal{O}(1), \quad|\omega| \rightarrow \infty, \quad \omega \in \mathbb{C} \backslash \Gamma .
$$


REMARK 3.6. The reduction to the Schrödinger operator $\mathbf{H}$ allow us to deduce lemma 3.5 from lemma 3.4 applying known results of Agmon [1] and Jensen-Kato $[7]$.

3.2. Time decay. In this section we combine spectral properties of the perturbed resolvent $\mathcal{R}(\omega)$ and the time decay for the unperturbed dynamics using the (finite) Born perturbation series. Denote by $\Sigma$ the set of eigenvalues of the operators $\mathcal{H}$. Lemma 3.1 imply that the eigenvalues cannot accumulate to the points $\pm m$ and then the set $\Sigma$ is finite. Our main result is the following.

TheOREM 3.7. Let conditions (1.5) and (3.1) hold. Then

$$
\left\|e^{-i t \mathcal{H}}-\sum_{\omega_{j} \in \Sigma} e^{-i \omega_{j} t} P_{j}\right\|_{\mathcal{L}\left(L_{\sigma}^{2}, L_{-\sigma}^{2}\right)}=\mathcal{O}\left(|t|^{-3 / 2}\right), \quad t \rightarrow \pm \infty
$$

with $\sigma>\frac{5}{2}$, where $P_{j}$ are the Riesz projections onto the corresponding eigenspaces of the operator $\mathcal{H}$.

Proof. By (3.7) it suffices to prove that for $\sigma>5 / 2$

$$
\left\|e^{-i t \tilde{\mathcal{H}}}-\sum_{\omega_{j} \in \Sigma} e^{-i \omega_{j} t} \tilde{P}_{j}\right\|_{\mathcal{L}\left(L_{\sigma}^{2}, L_{-\sigma}^{2}\right)}=\mathcal{O}\left(|t|^{-3 / 2}\right), \quad t \rightarrow \pm \infty
$$

where $\tilde{P}_{j}=C^{-1} P_{j} C$ are the Riesz projectors onto the corresponding eigenspaces of operator $\tilde{H}$. Lemma 3.5 and asymptotics (3.13) imply similarly to (2.16), that

$$
e^{-i t \tilde{\mathcal{H}}} \psi_{0}-\sum_{\omega_{j} \in \Sigma} e^{-i \omega_{j} t} \tilde{P}_{j} \psi_{0}=\frac{1}{2 \pi i} \int_{\Gamma} e^{-i \omega t}[\tilde{\mathcal{R}}(\omega+i 0)-\tilde{\mathcal{R}}(\omega-i 0)] \psi_{0} d \omega=\psi_{l}(t)+\psi_{h}(t)
$$

where

with a small $\delta>0$, and

$$
\tilde{P}_{j} \psi_{0}:=-\frac{1}{2 \pi i} \int_{\left|\omega-\omega_{j}\right|=\delta} \tilde{\mathcal{R}}(\omega) \psi_{0} d \omega
$$

$$
\begin{aligned}
& \psi_{l}(t)=\frac{1}{2 \pi i} \int_{\Gamma} l(\omega) e^{-i \omega t}[\tilde{\mathcal{R}}(\omega+i 0)-\tilde{\mathcal{R}}(\omega-i 0)] \psi_{0} d \omega, \\
& \psi_{h}(t)=\frac{1}{2 \pi i} \int_{\Gamma} h(\omega) e^{-i \omega t}[\tilde{\mathcal{R}}(\omega+i 0)-\tilde{\mathcal{R}}(\omega-i 0)] \psi_{0} d \omega
\end{aligned}
$$

where $l(\omega)$ and $h(\omega)$ are defined in Section 2.2. Further we analyze $\psi_{l}(t)$ and $\psi_{h}(t)$ separately.

3.2.1. Low energy decay. We consider the integral (3.22 over $(m, m+2 \varepsilon)$ only. The integral over $(-m-2 \varepsilon,-m)$ is dealt with in the same way. We prove the desired decay of $\psi_{l}(t)$ using a special case of lemma 10.2 from [7]. Denote by $\mathbf{B}$ a Banach space with the norm $\|\cdot\|$.

LEMmA 3.8. Let $F \in C([m, a], \mathbf{B})$, satisfy

$$
F(m)=F(a)=0,\left\|F^{\prime \prime}(\omega)\right\|=\mathcal{O}\left(|\omega-m|^{-3 / 2}\right), \omega \rightarrow m .
$$

Then

$$
\int_{m}^{a} e^{-i t \omega} F(\omega) d \omega=\mathcal{O}\left(t^{-3 / 2}\right), \quad t \rightarrow \infty
$$


Due to (3.13), we can apply lemma 3.8 with $F=l(\omega)(\tilde{\mathcal{R}}(\omega+i 0)-\tilde{\mathcal{R}}(\omega-i 0))$, $\mathbf{B}=\mathcal{L}\left(L_{\sigma}^{2}, L_{-\sigma}^{2}\right), a=m+2 \varepsilon$ with a small $\varepsilon>0$ and $\sigma>5 / 2$, to get

$$
\left\|\psi_{l}(t)\right\|_{L_{-\sigma}^{2}} \leq C(1+|t|)^{-3 / 2}\left\|\psi_{0}\right\|_{L_{\sigma}^{2}} \quad t \in \mathbb{R}, \quad \sigma>5 / 2
$$

3.2.2. High energy decay. The resolvents $R(\zeta), R_{0}(\zeta)$ are related by the Born perturbation series

$$
R(\zeta)=R_{0}(\zeta) I-R_{0}(\zeta) V R_{0}(\zeta)+R_{0}(\zeta) V R_{0}(\zeta) V R(\zeta), \quad \zeta \in \mathbb{C} \backslash[0, \infty)
$$

which follow by iteration of $R(\zeta)=R_{0}(\zeta) I-R_{0}(\zeta) V R(\zeta)$. Then by (3.12) we have

$$
\begin{aligned}
& \tilde{\mathcal{R}}(\omega)=\left(i \alpha \partial_{x}+\tilde{\mathcal{V}}(x)+\omega\right)\left[R_{0}\left(\omega^{2}-m^{2}\right) I-R_{0}\left(\omega^{2}-m^{2}\right) V R_{0}\left(\omega^{2}-m^{2}\right)\right. \\
& \left.+\quad R_{0}\left(\omega^{2}-m^{2}\right) V R_{0}\left(\omega^{2}-m^{2}\right) V R\left(\omega^{2}-m^{2}\right)\right] .
\end{aligned}
$$

Let us substitute series (3.28) into spectral representation $(3.23)$ for $\psi_{h}(t)$ :

$$
\begin{aligned}
& \psi_{h}(t)=\frac{1}{2 \pi i} \int_{\Gamma} e^{-i \omega t} h(\omega)\left(i \alpha \partial_{x}+\tilde{\mathcal{V}}(x)+\omega\right)\left[R_{0}\left(\zeta_{+}\right)-R_{0}\left(\zeta_{-}\right)\right] \psi_{0} d \omega \\
& +\frac{1}{2 \pi i} \int_{\Gamma} e^{-i \omega t} h(\omega)\left(i \alpha \partial_{x}+\tilde{\mathcal{V}}(x)+\omega\right)\left[R_{0}\left(\zeta_{+}\right) V R_{0}\left(\zeta_{+}\right)-R_{0}\left(\zeta_{-}\right) V R_{0}\left(\zeta_{-}\right)\right] \psi_{0} d \omega \\
& +\frac{1}{2 \pi i} \int_{\Gamma} e^{-i \omega t} h(\omega)\left(i \alpha \partial_{x}+\tilde{\mathcal{V}}(x)+\omega\right) \\
& \quad\left[R_{0}\left(\zeta_{+}\right) V R_{0}\left(\zeta_{+}\right) V R\left(\zeta_{+}\right)-R_{0}\left(\zeta_{-}\right) V R_{0}\left(\zeta_{-}\right) V R\left(\zeta_{-}\right)\right] \psi_{0} d \omega \\
& =\psi_{h 1}(t)+\psi_{h 2}(t)+\psi_{h 3}(t), \quad t \in \mathbb{R}
\end{aligned}
$$

where $\zeta_{+}=(\omega+i 0)^{2}-m^{2}, \zeta_{-}=(\omega-i 0)^{2}-m^{2}$. We analyze each term $\psi_{h k}$, $k=1,2,3$ separately.

Step i) For the first term $\psi_{h 1}(t)$ we have by $(2.5),(2.20)$ and $(2.22)$

$$
\psi_{h 1}(t)=-i(\tilde{\mathcal{V}}(x)-m \beta) \mathcal{G}_{h}(t) \psi_{0}+\mathcal{U}_{h}(t) \psi_{0} .
$$

Hence, (2.23) and (2.25) imply that

$$
\left\|\psi_{h 1}(t)\right\|_{L_{-\sigma}^{2}} \leq C(1+|t|)^{-3 / 2}\left\|\psi_{0}\right\|_{L_{\sigma}^{2}}, \quad t \in \mathbb{R}, \quad \sigma>5 / 2 .
$$

Step ii) We represent the second term $\psi_{h 2}(t)$ as

$$
\psi_{h 2}(t)=\varphi_{1}(t)+\varphi_{2}(t)
$$

where

$$
\begin{gathered}
\varphi_{1}(t)=\frac{1}{2 \pi i} \int_{\Gamma} e^{-i \omega t} h(\omega)(\tilde{\mathcal{V}}-m \beta)\left[R_{0}\left(\zeta_{+}\right) V R_{0}\left(\zeta_{+}\right)-R_{0}\left(\zeta_{-}\right) V R_{0}\left(\zeta_{-}\right)\right] \psi_{0} d \omega, \\
\varphi_{2}(t)=\frac{1}{2 \pi i} \int_{\Gamma} e^{-i \omega t} h(\omega)\left(i \alpha \partial_{x}+m \beta+\omega\right)\left[R_{0}\left(\zeta_{+}\right) V R_{0}\left(\zeta_{+}\right)-R_{0}\left(\zeta_{-}\right) V R_{0}\left(\zeta_{-}\right)\right] \psi_{0} d \omega \\
=\frac{1}{2 \pi i} \int_{\Gamma} e^{-i \omega t} h(\omega)\left[\mathcal{R}_{0}(\omega+i 0) V R_{0}\left(\zeta_{+}\right)-\mathcal{R}_{0}(\omega-i 0) V R_{0}\left(\zeta_{-}\right)\right] \psi_{0} d \omega .
\end{gathered}
$$

Consider the first term $\varphi_{1}(t)$. Denote

$$
P(\omega)=h(\omega)(\tilde{\mathcal{V}}-m \beta)\left[R_{0}\left(\zeta_{+}\right) V R_{0}\left(\zeta_{+}\right)-R_{0}\left(\zeta_{-}\right) V R_{0}\left(\zeta_{-}\right)\right] \psi_{0} .
$$


We have

$$
\operatorname{supp} P(\omega) \in \Gamma_{\varepsilon}:=(-\infty,-m-\varepsilon) \cup(m+\varepsilon, \infty),
$$

and $P^{\prime \prime} \in L^{1}\left(\Gamma_{\varepsilon} ; \mathcal{L}\left(L_{\sigma}^{2}, L_{-\sigma}^{2}\right)\right)$ with $\sigma>5 / 2$ by $(2.10)$ with $l=0$ and $k=2$ since $V$ does not contain differential operators, see Remark 3.3. Then, two times partial integration implies that

$$
\left\|\varphi_{1}(t)\right\|_{L_{-\sigma}^{2}} \leq C(1+|t|)^{-2}\left\|\psi_{0}\right\|_{L_{\sigma}^{2}}, \quad t \in \mathbb{R}, \quad \sigma>5 / 2
$$

Now we consider the second term $\varphi_{2}(t)$. Denote $h_{1}(\omega)=\sqrt{h(\omega)}$ (we can assume that $h(\omega) \geq 0$ and $\left.h_{1} \in \mathbb{C}_{0}^{\infty}(\mathbb{R})\right)$. We set

$$
\phi_{h 1}=\frac{1}{2 \pi i} \int_{\Gamma} e^{-i \omega t} h_{1}(\omega)\left[R_{0}\left(\zeta_{+}\right)-R_{0}\left(\zeta_{-}\right)\right] \psi_{0} d \omega=-i \mathcal{G}_{h_{1}}(t) \psi_{0}
$$

For $\phi_{h 1}$ inequality (3.29) obviously holds: Namely,

$$
\left\|\phi_{h 1}(t)\right\|_{L_{-\sigma}^{2}} \leq C(1+|t|)^{-3 / 2}\left\|\psi_{0}\right\|_{L_{\sigma}^{2}}, \quad t \in \mathbb{R}, \quad \sigma>5 / 2
$$

The term $\varphi_{2}(t)$ can be rewritten as a convolution.

Lemma 3.9. (cf. [9, Lemma 3.11]) The convolution representation holds

$$
\varphi_{2}(t)=i \int_{0}^{t} \mathcal{U}_{h_{1}}(t-\tau) V \phi_{h 1}(\tau) d \tau, t \in \mathbb{R}
$$

where the integral converges in $L_{-\sigma}^{2}$ with $\sigma>5 / 2$.

Applying theorem 2.3 with $h_{1}$ instead of $h$ to the integrand in (3.32), we obtain that

$$
\begin{aligned}
& \left\|\mathcal{U}_{h_{1}}(t-\tau) V \phi_{h 1}(\tau)\right\|_{L_{-\sigma}^{2}} \leq \frac{C\left\|V \phi_{h 1}(\tau)\right\|_{L_{\sigma^{\prime}}^{2}}}{(1+|t-\tau|)^{3 / 2}} \\
& \leq \frac{C_{1}\left\|\phi_{h 1}(\tau)\right\|_{L_{\sigma^{\prime}-\beta}^{2}} \leq \frac{C_{2}\left\|\psi_{0}\right\|_{L_{\sigma}^{2}}}{(1+|t-\tau|)^{3 / 2}} \leq \frac{\left.\right|^{3 / 2}(1+|\tau|)^{3 / 2}}{(1+|t-\tau|)^{3 / 2}}}{(1+)^{3}}
\end{aligned}
$$

where $\sigma^{\prime} \in(5 / 2, \beta-5 / 2)$. Therefore, integrating in $\tau$, we obtain by (3.32) that

$$
\left\|\varphi_{2}(t)\right\|_{L_{-\sigma}^{2}} \leq C(1+|t|)^{-3 / 2}\left\|\psi_{0}\right\|_{L_{\sigma}^{2}}, \quad t \in \mathbb{R}, \quad \sigma>5 / 2
$$

Step iii) Denote by $Q(\omega)$ the integrand in $\psi_{h 3}$. Since $Q^{\prime \prime} \in L^{1}\left(\Gamma_{\varepsilon} ; \mathcal{L}\left(L_{\sigma}^{2}, L_{-\sigma}^{2}\right)\right)$ with $\sigma>5 / 2$ by (2.10) and (3.15) then, two times partial integration implies that

$$
\left\|\psi_{h 3}(t)\right\|_{L_{-\sigma}^{2}} \leq C(1+|t|)^{-2}\left\|\psi_{0}\right\|_{L_{\sigma}^{2}}, \quad t \in \mathbb{R}, \quad \sigma>5 / 2 .
$$

Finally, bounds (3.29), (3.31), (3.34) and 3.35) imply

$$
\left\|\psi_{h}(t)\right\|_{L_{-\sigma}^{2}} \leq C(1+|t|)^{-3 / 2}\left\|\psi_{0}\right\|_{L_{\sigma}^{2}}, \quad t \in \mathbb{R}, \quad \sigma>5 / 2 .
$$

Theorem 3.7 is proved.

COROllary 3.10. Asymptotics (3.20) imply (1.6) with the projector

$$
\mathcal{P}_{c}:=1-\mathcal{P}_{d}, \quad \mathcal{P}_{d}=\sum_{\omega_{j} \in \Sigma} P_{j}
$$




\section{Application to asymptotic completeness}

We apply the obtained results to prove an asymptotic completeness by standard Cook's argument.

THEOREM 4.1. Let conditions (1.5) and (3.1) hold. Then

i) For the solution to (1.1) with the initial function $\psi(0) \in L^{2}$, the long time asymptotics hold

$$
\psi(t)=\sum_{\omega_{j} \in \Sigma} e^{-i \omega_{j} t} \psi_{j}+\mathcal{U}(t) \phi_{ \pm}+r_{ \pm}(t)
$$

where $\psi_{j}$ are the corresponding eigenfunctions, $\phi_{ \pm} \in L^{2}$ are the scattering states, and

$$
\left\|r_{ \pm}(t)\right\|_{L^{2}} \rightarrow 0, t \rightarrow \pm \infty
$$

ii) Furthermore,

$$
\left\|r_{ \pm}(t)\right\|_{L^{2}}=\mathcal{O}\left(|t|^{-1 / 2}\right)
$$

if $\psi(0) \in L_{\sigma}^{2}$ with $\sigma>5 / 2$.

Proof. Denote $\mathcal{X}_{d}:=\mathcal{P}_{d} L^{2}, \mathcal{X}_{c}:=\mathcal{P}_{c} L^{2}$. For $\psi(0) \in \mathcal{X}_{d}$ asymptotics (4.1) obviously hold with $\phi_{ \pm}=0$ and $r_{ \pm}(t)=0$. Hence, it remains to prove for $\psi(0) \in \mathcal{X}_{c}$ the asymptotics

$$
\psi(t)=\mathcal{U}(t) \phi_{ \pm}+r_{ \pm}(t)
$$

with the remainder satisfying (4.2). Moreover, it suffices to prove asymptotics (4.4), (4.3) for $\psi(0) \in \mathcal{X}_{c} \cap L_{\sigma}^{2}$ with $\sigma>5 / 2$ since the space $L_{\sigma}^{2}$ is dense in $L^{2}$, while the group $\mathcal{U}(t)$ is unitary in $L^{2}$. In this case theorem 3.7 implies the decay

$$
\|\psi(t)\|_{L_{-\sigma}^{2}} \leq C(1+|t|)^{-3 / 2}\|\psi(0)\|_{L_{\sigma}^{2}}, \quad t \rightarrow \pm \infty .
$$

The function $\psi(t)$ satisfies equation (1.1),

$$
i \dot{\psi}(t)=\left(\mathcal{H}_{0}+\mathcal{V}\right) \psi(t) .
$$

Hence, the corresponding Duhamel equation reads

$$
\psi(t)=\mathcal{U}(t) \psi(0)+\int_{0}^{t} \mathcal{U}(t-\tau) \mathcal{V} \psi(\tau) d \tau, t \in \mathbb{R}
$$

Rewrite (4.6) as

$\psi(t)=\mathcal{U}(t)\left[\psi(0)+\int_{0}^{ \pm \infty} \mathcal{U}(-\tau) \mathcal{V} \psi(\tau) d \tau\right]-\int_{t}^{ \pm \infty} \mathcal{U}(t-\tau) \mathcal{V} \psi(\tau) d \tau=\mathcal{U}(t) \phi_{ \pm}+r_{ \pm}(t)$.

It remains to prove that $\phi_{ \pm} \in L^{2}$ and (4.3) holds. Consider the sign "+" for the concreteness. The unitarity of $\mathcal{U}(t)$ in $L^{2}$, condition (1.5) and decay (4.5) imply 
that for $\sigma^{\prime} \in(5 / 2, \min \{\sigma, \beta\})$

$$
\begin{aligned}
& \int_{0}^{\infty}\|\mathcal{U}(-\tau) \mathcal{V} \psi(\tau)\|_{L^{2}} d \tau \leq C \int_{0}^{\infty}\|\mathcal{V} \psi(\tau)\|_{L^{2}} d \tau \\
& \leq C_{1} \int_{0}^{\infty}\|\psi(\tau)\|_{L_{-\sigma^{\prime}}^{2}} d \tau \leq C_{2} \int_{0}^{\infty} \frac{\|\psi(0)\|_{L_{\sigma}^{2}} d \tau}{(1+\tau)^{-3 / 2}}<\infty
\end{aligned}
$$

since $|\mathcal{V}(x)| \leq C\langle x\rangle^{-\beta} \leq C^{\prime}\langle x\rangle^{-\sigma^{\prime}}$. Hence, $\phi_{+} \in L^{2}$. Estimate (4.3) follows similarly.

\section{References}

[1] Agmon S., Spectral properties of Schrödinger operator and scattering theory, Ann. Scuola Norm. Sup. Pisa, Ser. IV 2, 151-218 (1975).

[2] Bleher P.M., On operators depending meromorphically on a parameter, Moscow Univ. Math. Bull. 24, 21-26 (1972).

[3] N. Boussaid, Stable directions for small nonlinear Dirac standing waves, Comm. Math. Phys. 268 (2006), no. 3, 757-817.

[4] Gohberg I.C., Krein M.G., Introduction to the Theory of Linear Nonselfadjoint Operators, American Mathematical Society, Providence, RI, 1969.

[5] Jensen A., Spectral properties of Schrödinger operators and time-decay of the wave function. Results in $L^{2}\left(\mathbb{R}^{m}\right), m \geq 5$, Duke Math. J. 47, 57-80 (1980).

[6] Jensen A., Spectral properties of Schrödinger operators and time-decay of the wave function. Results in $L^{2}\left(\mathbb{R}^{4}\right)$, J. Math. Anal. Appl 101, 491-513 (1984).

[7] Jensen A., Kato T., Spectral properties of Schrödinger operators and time-decay of the wave functions, Duke Math. J. 46, 583-611 (1979)

[8] Jensen A., Nenciu G., A unified approach to resolvent expansions at thresholds, Rev. Math. Phys. 13, No.6, 717-754 (2001).

[9] Komech A., Kopylova E., Weighted energy decay for 1D Klein-Gordon equation, Comm. PDE 35, no.2, 353-374 (2010).

[10] Komech A., Kopylova E., Long time decay for 2D Klein-Gordon equation, J. Funct. Analysis 259 (2010), no. 2, 477-502.

[11] Komech A., Kopylova E., Weighted energy decay for 3D Klein-Gordon equation, J. Diff. Eqns. 248 (2010), no. 3, 501-520.

[12] Murata M., Asymptotic expansions in time for solutions of Schrödinger-type equations, J. Funct. Anal. 49, 10-56 (1982).

[13] Watson G.N., Bessel Functions, Cambridge, 1922.

Institute for Information Transmission Problems RAS, B.Karetnyi 19, Moscow 101447,GSP-4, RUSSIA

E-mail address: elena.kopylova@univie.ac.at 